\title{
Förderung des wissenschaftlichen Nachwuchses - auch Ziel einer evidenzbasierten und wissenschaftlich fundierten Fortbildung
}

Dies ist nicht das erste Editorial, in dem auf die Bedeutung der Förderung des wissenschaftlichen Nachwuchses in der Inneren Medizin hingewiesen wird. Diese Förderaktivitäten sind auch vor dem Hintergrund von überaus großer Bedeutung, dass wir zunehmend einen Personalmangel im stationären wie im ambulanten Bereich erleben - dies gilt auch für die Innere Medizin. Die Gründe hierfür sind vielfältig, sie beginnen ohne Frage mit der Berufswahl in der Medizin und der Kontinuität der Berufsausübung. Wir müssen hier sicher zwischen einem relativen und absoluten Ärztemangel unterscheiden; kurz gefasst ergibt sich ein absoluter Ärztemangel durch z.B. die Entscheidung, keine Weiterbildung zum Facharzt aufzunehmen, aufgrund der demographischen Entwicklung, gesetzlicher Veränderungen wie Arbeitszeitgesetz und auch die - ohne Einschränkung wichtige - Feminisierung der Medizin. Gründe für einen relativen Ärztemangel sind in einer erheblichen Verdichtung der Arbeitsinhalte zu sehen; so steigt in hohem Maße die Zahl geriatrischer Patienten mit den für ältere Menschen typischen Komorbiditäten, und der Krankheitsverlauf von vielen Patienten nimmt erfreulicherweise - eine chronische Entwicklung (Beispiel: Tumorpatienten).

Diese in allen Bereichen der Medizin notwendige qualitative und quantitative Zunahme an diagnostischen und therapeutischen Maßnahmen bedingt natürlich automatisch, dass bei gleichbleibender Anzahl der Ärzte eine relative Unterversorgung entsteht. Von dem daraus resultierenden Nachwuchsmangel ist auch die klinische Forschung betroffen; wir hatten uns von Seiten der Deutschen Gesellschaft für Innere Medizin mehrfach und vehement dafür eingesetzt, dass klare Regeln für die wissenschaftliche Arbeit von motivierten und ehrgeizigen jungen Ärztinnen und Ärzten geschaffen werden müssen. Die Deutsche Gesellschaft für Innere Medizin hat sich klar dahingehend positioniert, dass die wissenschaftliche Arbeit eindeutig derselben Wertschätzung wie die klinische bedarf. Für die $\mathrm{Zu}-$ kunft wird es daher eine wesentliche Aufgabe sein, Komplementarität und gegenseitige Angewiesenheit beider Ebenen klar zu stellen.
Auch in unseren Jahreskongressen wird der wissenschaftliche Nachwuchs z.B. durch die Integration der Forschungsergebnisse in den Kongress und Auszeichnungen herausragender Leistungen durch Preise und Stipendien gefördert.

Klinische Forschung in der Inneren Medizin wird und kann aber nur dann erfolgreich sein, wenn die Innere Medizin in ihrer Gesamtheit auch in ihren Schwerpunkten unvermindert gestärkt und diese adäquat an Universitätskliniken und Schwerpunkt-Krankenhäusern repräsentiert sind. Die enge Zusammenarbeit mit den Schwerpunktgesellschaften der Inneren Medizin ist daher für die DGIM eine selbstverständliche Aufgabe.

Und schließlich: Förderung des klinischen und wissenschaftlichen Nachwuchses muss auch übergeordnetes Ziel einer evidenzbasierten und wissenschaftlich fundierten Fortbildung sein. Genau dieses Ziel verfolgen auch die DGIM-Gesellschaftsausgaben der DMW: durch wissenschaftliche Fortbildungsartikel auf höchstem Niveau und mit dem Ziel, die Innere Medizin nicht nur in ihrer Gesamtheit, sondern auch gegenseitigen Bedingtheit darzustellen. Wir sind davon überzeugt, dass auch dies dazu beiträgt, das Fach Innere Medizin weiterhin als ein hoch attraktives für den Nachwuchs zu vermitteln und zu gestalten.

Ohne Frage ist das auch mit den Artikeln in diesem Heft wieder gelungen; in jedem einzelnen Artikel wird deutlich, dass zur rationalen und erfolgreichen Diagnostik und Therapie eines Krankheitsbildes die Kenntnis seiner Verbindungen $\mathrm{zu}$ zahlreichen anderen internistischen Krankheitsbildern (und eben auch anderen Schwerpunkten) gehört.

In diesem Sinne wünsche ich der Ausgabe „Klinischer Fortschritt“ 2/2012 viel Erfolg und große Resonanz!

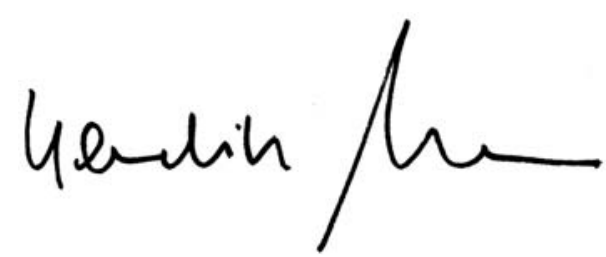

Prof. Dr. med. Hendrik Lehnert

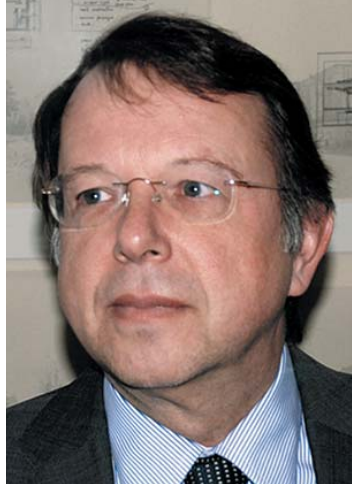

H. Lehnert

Innere Medizin

Institut

Medizinische Klinik I, Universitätsklinikum Schleswig-Holstein, Campus Lübeck

Bibliografie

DOI 10.1055/s-0031-1299027 Dtsch Med Wochenschr 2012; 137: 621 . (c) Georg Thieme Verlag KG · Stuttgart · New York - ISSN 0012-0472

Korrespondenz Prof. Dr. Dr. h.c. Hendrik Lehnert Medizinische Klinik 1, Universitätsklinikum SchleswigHolstein, Campus Lübeck Ratzeburger Allee 160 23538 Lübeck Tel. 0451/500-2306 Fax 0451/500-3339 